\title{
HOMOTOPY THEORY OF HOPF GALOIS EXTENSIONS
}

\author{
CHRISTIAN KASSEL AND HANS-JÜRGEN SCHNEIDER
}

\begin{abstract}
We introduce the concept of homotopy equivalence for Hopf Galois extensions and make a systematic study of it. As an application we determine all $H$-Galois extensions up to homotopy equivalence in the case when $H$ is a Drinfeld-Jimbo quantum group.
\end{abstract}

KEy Words: Galois extension, Hopf algebra, quantum group, homotopy, noncommutative geometry, principal fibre bundle

\section{TITRE : Homotopie des extensions de Hopf Galois}

RÉSumÉ. Nous étudions le concept d'équivalence d'homotopie pour les extensions $H$-galoisiennes où $H$ désigne une algèbre de Hopf. Ceci nous permet de classifier les extensions H-galoisiennes à homotopie près lorsque $H$ est un groupe quantique de Drinfeld-Jimbo.

MotS-CLÉS: extension galoisienne, algèbre de Hopf, groupe quantique, homotopie, géométrie non commutative, fibré principal

Mathematics Subject Classification (2000): 16W30, 17B37, 55R10, 58B34, 81R50, 81R60

\section{INTRODUCTION}

The purpose of this article is to make a systematic study of the concept of homotopy equivalence introduced in the framework of Hopf Galois extensions by the first-named author [K]. As has been stressed many times (see e.g. [S] ), Hopf Galois extensions can be viewed as noncommutative analogues of principal fibre bundles where the role of the structural group is played by a Hopf algebra. It is therefore natural to adapt the concept of homotopy to them. 
Hopf Galois extensions for a given Hopf algebra and over a given algebra are difficult to classify up to isomorphism. One of our motivations in this paper and in [K] was that it might be easier to classify Hopf Galois extensions up to homotopy equivalence. We show in this paper that it is indeed so in the case when $H$ is a Drinfeld-Jimbo quantum group or some finite-dimensional variant. More precisely, we prove that the homotopy classes of $H$-Galois extensions for such a Hopf algebra $H$ are in bijection with the homotopy classes of $k[G]$-Galois extensions, where $G$ is the group of group-like elements of $H$.

Certain $K$-theoretic elements naturally attached to Hopf Galois extensions and recently investigated in connection with non-commutative geometry (see the survey article $[\mathrm{BH}]$ and references therein) turn out to be homotopy invariant. This gives another reason to consider homotopy equivalence in this algebraic framework.

In Section 1 we collect basic definitions and various results in the literature in order to state functorial properties of the set $\operatorname{Gal}_{B}(H)$ of isomorphism classes of faithfully flat $H$-Galois extensions of an algebra $B$. This includes change of scalars, change of Hopf algebras, and twistings of Galois extensions.

Section 2 explains the concept of homotopy equivalence of Hopf Galois extensions, which had been introduced in $[\mathrm{K}$ for central extensions (contrary to loc. cit., we avoid here any reference to étale morphisms). Our most striking result on the set $\mathcal{H}_{B}(H)$ of homotopy classes of faithfully flat $H$-Galois extensions of an algebra $B$ is the following: if $H=\oplus_{n \geq 0} H(n)$ is an $\mathbb{N}$-graded Hopf algebra, then the inclusion $H(0) \subset H$ induces a bijection

$$
\mathcal{H}_{B}(H) \cong \mathcal{H}_{B}(H(0)) .
$$

In Section 3 we make the connection between our definition of homotopy equivalence and the construction of homotopy functors in algebraic $K$-theory from which it is directly inspired. When $B$ is a commutative ring satisfying certain conditions and $H=k[G]$ is a group algebra, we relate $\operatorname{Gal}_{B}(H)$ and $\mathcal{H}_{B}(H)$ to the Picard group $\operatorname{Pic}(B)$ and some cohomology group of $G$.

Finally, in Section 4 we show that, when $U_{q}(\mathfrak{g})$ is a Drinfeld-Jimbo quantum group and $G$ is the group of its group-like elements, then

$$
\mathcal{H}_{B}\left(U_{q}(\mathfrak{g})\right) \cong \mathcal{H}_{B}(k[G]) .
$$

This follows from the results obtained in the previous sections and from the fact that $U_{q}(\mathfrak{g})$ is a twist of a graded Hopf algebra whose component of degree 0 is $k[G]$ (for results related to the latter fact, see [AS], [Di]). 
Throughout the paper, the ground ring is an arbitrary commutative ring $k$. Unadorned tensor product means tensor product over $k$, and algebras and coalgebras are defined over $k$. We denote by $U(R)$ the group of invertible elements of a ring $R$, by $G(H)$ the group of grouplike elements of a Hopf algebra $H$, and by $C_{n}$ the cyclic group of order $n$.

If $C$ is a coalgebra, and $V$ a right (resp. left) $C$-comodule, we use the following version of the Sweedler notation: $\Delta(c)=c_{(1)} \otimes c_{(2)}$ for the comultiplication of $c \in C$, and $v_{(0)} \otimes v_{(1)}\left(\right.$ resp. $\left.v_{(-1)} \otimes v_{(0)}\right)$ for the coaction of $v \in V$.

\section{Hopf Galois extensions}

Let $H$ be a Hopf algebra, and $A$ a right $H$-comodule algebra with structure map $\delta: A \rightarrow A \otimes H, a \mapsto \delta(a)=a_{(0)} \otimes a_{(1)}$, that is, $\delta$ is an algebra map and a right $H$-comodule structure. The subalgebra $A^{\mathrm{co} H} \subset A$ of $H$-coinvariant elements is defined as

$$
A^{\mathrm{co} H}=\left\{a \in A \mid a_{(0)} \otimes a_{(1)}=a \otimes 1\right\} .
$$

Let $B$ be an algebra. A (right) $H$-comodule algebra over $B$ is a triple $(A, \delta, i)$, where $A$ is a right $H$-comodule algebra with structure map $\delta$ and $i: B \rightarrow A$ is an algebra map with $i(B) \subset A^{\mathrm{co} H}$. For any $H$ comodule algebra $(A, \delta, i)$ over $B$, the canonical or Galois map is defined by

$$
\text { can }: A \otimes_{B} A \rightarrow A \otimes H, x \otimes y \mapsto x y_{(0)} \otimes y_{(1)} .
$$

An $H$-comodule algebra $(A, \delta, i)$, or simply $A$ over $B$, is an $H$-Galois extension of $B$ if its Galois map is bijective and if $i$ defines an isomorphism $i: B \rightarrow A^{\mathrm{co} H}$. If $A$ is a right $H$-comodule algebra and $B$ is a subalgebra of $A$, we will say that $B \subset A$ is an $H$-Galois extension if $B=A^{\mathrm{co} H}$ and if the Galois map of $A$ over $B$ is bijective.

An $H$-comodule algebra $A$ is called $H$-cleft if there exists a right $H$-colinear map $\gamma: H \rightarrow A$ that is invertible up to convolution. If $A$ is $H$-cleft, then $A^{\mathrm{co} H} \subset A$ is $H$-Galois (see [M, 8.2.4]).

A morphism $\varphi:(A, \delta, i) \rightarrow(\widetilde{A}, \widetilde{\delta}, \widetilde{i})$ of $H$-comodule algebras over $B$ is a right $H$-colinear algebra map $\varphi: A \rightarrow \widetilde{A}$ with $\widetilde{i}=\varphi i$. An isomorphism of $H$-comodule algebras is a bijective morphism. Note that an $H$-colinear algebra isomorphism of $H$-Galois extensions of $B$ is an isomorphism of comodule algebras over $B$.

Lemma 1.1. Let $H$ be a Hopf algebra, $B$ be an algebra, and $(A, \delta, i)$ and $(\widetilde{A}, \widetilde{\delta}, \widetilde{i})$ be $H$-comodule algebras over $B$ with bijective Galois maps.

(1) [T, 4.2] Assume that $A$ is faithfully flat as a right or left $B$ module via $i$. Then $A$ is an $H$-Galois extension of $B$. 
(2) [S. 3.11(1)] Let $\varphi: A \rightarrow \widetilde{A}$ be a morphism of $H$-comodule algebras over $B$. Assume that $\widetilde{A}$ is faithfully flat as a right $B$ module via $\widetilde{i}$. Then $\varphi$ is an isomorphism, and both $A$ and $\widetilde{A}$ are $H$-Galois extensions of $B$.

To define homotopy (see Section 2) we have to extend the ground ring from $k$ to the polynomial algebra $k[t]$ in the indeterminate $t$.

In general, let $\alpha: k \rightarrow R$ be a homomorphism of commutative rings. Ground ring extension from $k$ to $R$ then means tensoring with $R$ over $k$, where $R$ is a module over $k$ via $\alpha$. If $B$ is a $k$-algebra and $M$ a left $B$ module, then $R \otimes B$ is an $R$-algebra, and $R \otimes M$ is a left $R \otimes B$-module in the natural way by

$$
(r \otimes b)(s \otimes m)=r s \otimes b m
$$

for all $r, s \in R, b \in B, m \in M$. Recall that for any right $R \otimes B$ module $X$,

$$
X \otimes_{R \otimes B}(R \otimes M) \rightarrow X \otimes_{B} M, x \otimes r \otimes m \mapsto x r \otimes m,
$$

is an isomorphism. In particular, ground ring extension preserves flatness and faithful flatness. Similarly, if $C$ is a $k$-coalgebra and $V$ a right $C$-comodule, then $R \otimes C$ is an $R$-coalgebra and $R \otimes V$ is a right $R \otimes C$-comodule. Note that a right $R \otimes C$-comodule structure on an $R$-module $W$ is an $R$-linear right $C$-comodule structure

$$
W \rightarrow W \otimes_{R}(R \otimes C) \cong W \otimes C .
$$

If $A$ is an $H$-comodule algebra over $B$, then we obtain by ground ring extension an $R \otimes H$-comodule algebra $R \otimes A$ over $R \otimes B$ with ground ring $R$.

Proposition 1.2. Let $H$ be a Hopf algebra, $B$ an algebra, and $A$ an $H$ Galois extension of $B$ with ground ring $k$. Assume that $A$ is left (resp. right) faithfully flat over $B$. Let $\alpha: k \rightarrow R$ be a homomorphism of commutative rings. Then $R \otimes A$ is an $R \otimes H$-Galois extension of $R \otimes B$, and $R \otimes A$ is left (resp. right) faithfully flat over $R \otimes B$.

Proof. It is clear that the Galois map of $R \otimes A$ is bijective and that $R \otimes A$ is left (resp. right) faithfully flat over $R \otimes B$. Hence the claim follows from Lemma 1.1 (1).

The functorial behaviour of $H$-Galois extensions or $H$-comodule algebras in the Hopf algebra $H$ as a variable is given by the cotensor product. If $C$ is a coalgebra, and $V$ and $W$ are right and left $C$-comodules 
with comodule structures $\delta_{V}: V \rightarrow V \otimes C$ and $\delta_{W}: W \rightarrow C \otimes W$, then the cotensor product $V \square_{C} W$ is the kernel of

$$
\delta_{V} \otimes \mathrm{id}_{W}-\mathrm{id}_{V} \otimes \delta_{W}: V \otimes W \rightarrow V \otimes C \otimes W .
$$

Let $\varphi: K \rightarrow H$ be a Hopf algebra homomorphism, and $A$ a right $H$ comodule algebra over $B$ with algebra map $i: B \rightarrow A$. Assume that $K$ is flat as a $k$-module. Then $A \square_{H} K \subset A \otimes K$ is a subalgebra and right $K$-subcomodule of $A \otimes K$ with componentwise multiplication and $K$-comodule structure $\operatorname{id}_{A} \otimes \Delta$. Here we view $K$ as a left $H$-comodule by

$$
K \rightarrow H \otimes K, x \mapsto \varphi\left(x_{(1)}\right) \otimes x_{(2)} .
$$

Note that the inclusion $A \square_{H} K \subset A \otimes K$ defines an injective map

$$
\left(A \square_{H} K\right) \otimes K \rightarrow A \otimes K \otimes K
$$

since $K$ is flat over $k$. Hence the $K$-comodule structure of $A \square_{H} K$ is well defined.

Moreover, $A \square_{H} K$ is a $K$-comodule algebra over $B$ by the algebra $\operatorname{map} B \rightarrow A \square_{H} K, b \mapsto i(b) \otimes 1$.

Proposition 1.3. [S, 3.11 (3)] Let $\varphi: K \rightarrow H$ be a Hopf algebra homomorphism and $A$ an $H$-Galois extension of $B$. Assume that $A$ is right faithfully flat over $B$ and that $K$ is flat over $k$. Then $A \square_{H} K$ is a K-Galois extension of $B$ and right faithfully flat over $B$.

Let $H$ be a Hopf algebra and $B$ an algebra over $k$. We denote by $\operatorname{Gal}_{B}(H / k)$, or simply by $\operatorname{Gal}_{B}(H)$, the set of isomorphism classes of $H$-Galois extensions of $B$ that are right faithfully flat over $B$.

Let $\alpha: R \rightarrow S$ be a homomorphism of commutative $k$-algebras. For any right faithfully flat $R \otimes H$-Galois extension $A$ of $R \otimes B$ let $\alpha_{*} A=S \otimes_{R} A$ be the $S \otimes H$-Galois extension of $S \otimes B$ defined by ground ring extension, where we identify

$$
S \otimes_{R}(R \otimes H) \cong S \otimes H \text { and } S \otimes_{R}(R \otimes B) \cong S \otimes B .
$$

Let $\varphi: K \rightarrow H$ a Hopf algebra homomorphism. Assume that $K$ is flat over $k$. For any right faithfully flat $H$-Galois extension $A$ of $B$ let $\varphi^{*} A=A \square_{H} K$ be the $K$-Galois extension of $B$ given by the cotensor product.

Then $\alpha_{*}$ and $\varphi^{*}$ define maps

$$
\begin{aligned}
\alpha_{*}: \mathrm{Gal}_{R \otimes B}(R \otimes H) & \rightarrow \mathrm{Gal}_{S \otimes B}(S \otimes H), \\
\varphi^{*}: \mathrm{Gal}_{B}(H) & \rightarrow \mathrm{Gal}_{B}(K) .
\end{aligned}
$$

We collect the basic rules concerning ()$^{*}$ and ()$_{*}$. In particular, $\operatorname{Gal}_{R \otimes B}(R \otimes H)$ is a covariant functor in commutative $k$-algebras $R$ and $\operatorname{Gal}_{B}(H)$ is a contravariant functor in $k$-flat Hopf algebras $H$. 
Proposition 1.4. Let $H$ be a Hopf algebra over $k$ and $A$ a right faithfully flat $H$-Galois extension of $B$. Let $\alpha: k \rightarrow R$ and $\beta: R \rightarrow S$ be homomorphisms of commutative $k$-algebras, $K, L$ be $k$-flat Hopf algebras, and $\psi: L \rightarrow K, \varphi: K \rightarrow H$ be Hopf algebra homomorphisms. Then

(1) $(\alpha \beta)_{*} A \cong \alpha_{*} \beta_{*} A$.

(2) $(\varphi \psi)^{*} A \cong \psi^{*} \varphi^{*} A$.

(3) $\alpha_{*} \varphi^{*} A \cong\left(\operatorname{id}_{R} \otimes \varphi\right)^{*} \alpha_{*} A$.

Proof. (1) follows from the associativity of the tensor product.

To prove (2), we first define a right $L$-colinear algebra map

$$
f: A \otimes L \rightarrow A \otimes K \otimes L, a \otimes x \mapsto a \otimes \psi\left(x_{(1)}\right) \otimes x_{(2)} .
$$

The defining sequence

$$
0 \rightarrow A \square_{H} K \rightarrow A \otimes K \rightarrow A \otimes H \otimes K
$$

of $\varphi^{*} A=A \square_{H} K$ remains exact after tensoring with $L$ over $k$ since $L$ is $k$-flat. Then we see that $f$ maps the subspace $(\varphi \psi)^{*} A=A \square_{H} L$ into $\left(A \square_{H} K\right) \otimes L$. Finally, $f\left(A \square_{H} L\right)$ is contained in $\psi^{*} \varphi^{*} A=\left(A \square_{H} K\right) \square_{K} L$ by the definition of the cotensor product of $A \square_{H} K$ with $L$. Note that we have used flatness of $K$ over $k$ to define the $k$-comodule structure of $A \square_{H} K$.

Thus we have defined a right $L$-colinear algebra map

$$
A \square_{H} L \rightarrow\left(A \square_{H} K\right) \square_{K} L
$$

which is the identity on the subalgebra $B$ embedded in the first factor. By Lemma 1.1 (2) and Proposition 1.3 this map is an isomorphism, and (2) is proved.

(3) By Propositions 1.2 and Proposition 1.3.

$$
\alpha_{*} \varphi^{*} A=R \otimes\left(A \square_{H} K\right) \text { and }\left(\operatorname{id}_{R} \otimes \varphi\right)^{*} \alpha_{*} A=(R \otimes A) \square_{R \otimes H}(R \otimes K)
$$

are both right faithfully flat $R \otimes K$-Galois extensions of $R \otimes B$. Since the map

$$
R \otimes\left(A \square_{H} K\right) \rightarrow(R \otimes A) \square_{R \otimes H}(R \otimes K)
$$

defined by $r \otimes \sum_{i} a_{i} \otimes x_{i} \mapsto \sum_{i} r \otimes a_{i} \otimes 1 \otimes x_{i}$ is well defined and a morphism of $R \otimes K$-Galois extension of $R \otimes B$, it is bijective by Lemma $1.1(2)$.

Let $H$ be a Hopf algebra and $R$ a commutative algebra. An $H$-Galois extension $(A, \delta, i)$ of $R$ is called central if $i(R)$ is contained in the center of $A$. We denote by $\mathrm{CGal}_{R}(H / k)$, or simply by $\mathrm{CGal}_{R}(H)$, the set of isomorphism classes of faithfully flat central $H$-Galois extensions of $R$. 
Faithfully flat $H$-Galois extensions of the ground ring $k$ are also called $H$-Galois objects.

Remark 1.5. If $R \subset A$ is an $H$-Galois extension and if $R$ is central in $A$, then $A$ is an $R$-algebra and we can view $R \subset A$ as an $R \otimes H$ Galois extension of $R$ over the ground ring $R$ with comodule algebra structure

$$
A \rightarrow A \otimes H \cong A \otimes_{R}(R \otimes H) .
$$

Thus central Galois extensions of $R$ can be identified with Galois objects and there is a functorial isomorphism

$$
R \mapsto \operatorname{Gal}_{R}(R \otimes H / R) \cong \mathrm{CGal}_{R}(H / k)
$$

Finally we briefly recall the notion of twisting of Hopf algebras and comodule algebras [D, Theorem 1.6], [KS, 10.2.3]. Let $H$ be a Hopf algebra and $\sigma: H \otimes H \rightarrow k$ be a (normalized) 2-cocycle, that is,

$$
\sigma\left(x_{(1)}, y_{(1)}\right) \sigma\left(x_{(2)} y_{(2)}, z\right)=\sigma\left(y_{(1)}, z_{(1)}\right) \sigma\left(x, y_{(2)} z_{(2)}\right)
$$

for all $x, y, z \in H$, and $\sigma(x, 1)=\varepsilon(x)=\sigma(1, x)$.

Assume that $\sigma$ is invertible with inverse $\sigma^{-1}$ with respect to the convolution product. Then the twisted Hopf algebra $H^{\sigma}$ is $H$ as a coalgebra with the twisted multiplication

$$
x \cdot{ }_{\sigma} y=\sigma\left(x_{(1)}, y_{(1)}\right) x_{(2)} y_{(2)} \sigma^{-1}\left(x_{(3)}, y_{(3)}\right) .
$$

Let $A$ be a right $H$-comodule algebra. Then the twisted $H^{\sigma}$-comodule algebra $A^{\sigma}$ is $A$ as an $H$-comodule with the twisted multiplication

$$
a \cdot{ }_{\sigma} b=a_{(0)} b_{(0)} \sigma^{-1}\left(a_{(1)}, b_{(1)}\right) .
$$

The convolution inverse $\sigma^{-1}$ of $\sigma$ is an invertible 2-cocycle for $H^{\sigma}$, and $\left(A^{\sigma}\right)^{\sigma^{-1}}=A$ as a comodule algebra over $\left(H^{\sigma}\right)^{\sigma^{-1}}=H$.

Proposition 1.6. Let $H$ be a Hopf algebra, $\sigma: H \otimes H \rightarrow k$ an invertible 2-cocycle, and $B$ an algebra.

(1) If $A$ is an $H$-Galois extension of $B$, then $A^{\sigma}$ is an $H^{\sigma}$-Galois extension of $B$.

(2) The map $\operatorname{Gal}_{B}(H) \rightarrow \operatorname{Gal}_{B}\left(H^{\sigma}\right)$ given by $A \mapsto A^{\sigma}$ is bijective.

Proof. (1) is shown in [MS, Section 4], and (2) follows from (1) since $\left(A^{\sigma}\right)^{\sigma^{-1}}=A$. 


\section{2. Номотору}

The homotopy properties of principal bundles in topology are based on the following result. Let $G$ be a topological group, $B$ a topological space, and $\xi$ a numerable principal $G$-bundle over $B$. If $f_{i}: B^{\prime} \rightarrow B$, $i=0,1$, are homotopic maps, then the pull-back bundles $f_{0}^{*}(\xi)$ and $f_{1}^{*}(\xi)$ are isomorphic $\mathrm{Hu}$, Chapter 4, Theorem 9.9].

An $H$-Galois extension $B \subset A$ can be viewed as the algebraic analogue of the function algebra of a principal $G$-bundle $p: X \rightarrow B$. To study the algebraic analogue of homotopy for Hopf Galois extension we first recall the definition of homotopic algebra maps used in algebraic $K$-theory (see G, Section 3], [Sw1, Section 4]).

Let $k \subset k[t]$ be the polynomial algebra in the indeterminate $t$. For any $k$-module $V$, we denote the ground ring extension with respect to $k \subset k[t]$ by $V[t]$. We define $k$-linear maps $V[i]: V[t] \rightarrow V$ for $i \in\{0,1\}$ by sending $v t^{n}$ to $v i^{n}$. We will usually write simply $[i]$ instead of $V[i]$. Note that these maps are algebra maps if $V$ is an algebra.

Definition 2.1. Let $\alpha, \beta: R \rightarrow S$ be homomorphisms of commutative $k$-algebras. We say that $\alpha$ and $\beta$ are homotopic $(\alpha \sim \beta)$ if there exists a $k$-algebra homomorphism $\theta: R \rightarrow S[t]$ such that

$$
[0] \theta=\alpha \text { and }[1] \theta=\beta \text {. }
$$

The map $\theta$ is called a homotopy between $\alpha$ and $\beta$. Let $\approx$ be the equivalence relation generated by $\sim$.

The homomorphism $\alpha: R \rightarrow S$ is a homotopy equivalence if there exists a ring homomorphism $\alpha^{\prime}: S \rightarrow R$ with $\alpha \alpha^{\prime} \approx \operatorname{id}_{S}$ and $\alpha^{\prime} \alpha \approx \operatorname{id}_{R}$.

Remarks 2.2. (1) The relation $\sim$ is reflexive and symmetric (as one sees by the mapping $t \mapsto t-1)$. Thus $\alpha \approx \beta$ means that there is a sequence of algebra homomorphisms $\alpha_{1}, \ldots, \alpha_{n}: R \rightarrow S$ with $\alpha_{1}=\alpha$, $\alpha_{1} \sim \alpha_{2}, \ldots, \alpha_{n-1} \sim \alpha_{n}$, and $\alpha_{n}=\beta$.

(2) Let $R=\oplus_{n \geq 0} R(n)$ be an $\mathbb{N}$-graded commutative algebra. Then the inclusion $R(0) \subset R$ is a homotopy equivalence of commutative rings. We recall the argument in $[\mathrm{K}]$. Let $\iota: R(0) \rightarrow R$ be the inclusion, and $\pi: R \rightarrow R(0)$ the projection given by the grading. Then $\iota$ and $\pi$ are ring homomorphisms, and $\pi \iota=\mathrm{id}_{R(0)}$. We define an additive map $\theta: R \rightarrow R[t]$ by $\theta(x)=t^{n} x$ if $x \in R(n)(n \geq 0)$. Since $R$ is $\mathbb{N}$-graded, $\theta$ is a ring homomorphism. By construction, $[0] \theta=\iota \pi$ and $[1] \theta=\mathrm{id}_{R}$, hence $\iota \pi \sim \mathrm{id}_{R}$.

(3) Here is a non-graded example of a homotopy equivalence. Let $k$ be a field of characteristic $p>0$ and $k[G]$ the group algebra of a finite abelian $p$-group $G$. Write $G$ as a direct product of cyclic $p$-groups with 
generators $g_{i}, 1 \leq i \leq r$. Then the algebra map

$$
\theta: k[G] \rightarrow k[G][t] \text { with } \theta\left(g_{i}\right)=1+t\left(g_{i}-1\right), 1 \leq i \leq r,
$$

is a homotopy with $[0] \theta\left(g_{i}\right)=1$ and $[1] \theta\left(g_{i}\right)=g_{i}$ for all $i$. Hence the inclusion $k \subset k[G]$ is a homotopy equivalence.

Definition 2.3. Let $H$ be a Hopf algebra and $B$ an algebra. Let $A_{0}$ and $A_{1}$ be right faithfully flat $H$-Galois extensions of $B$. We write $A_{0} \sim A_{1}$ if there exists a right faithfully flat $H[t]$-Galois extension $A$ of $B[t]$ with ground ring $k[t]$ such that $[i]_{*} A \cong A_{i}$ for $i \in\{0,1\}$.

Homotopy equivalence of right faithfully flat $H$-Galois extensions of $B$ is the equivalence relation $\approx$ generated by $\sim$.

Let $\mathcal{H}_{B}(H / k)$, or simply $\mathcal{H}_{B}(H)$, be the set of homotopy equivalence classes of right faithfully flat $H$-Galois extensions of $B$.

Remarks 2.4. (1) Homotopy of $H$-Galois extensions can be formulated without changing the ground ring from $k$ to $k[t]$. Let $A_{0}$ and $A_{1}$ be right faithfully flat $H$-Galois extensions of $B$. Then the following are equivalent:

(a) $A_{0} \sim A_{1}$.

(b) There is a right faithfully flat $H$-Galois extension $A$ of $B[t]$ with ground ring $k$ such that $t$ lies in the center of $A$ and

$$
A /(t-i) \cong A_{i}
$$

as $H$-Galois extensions of $B$ for $i \in\{0,1\}$.

The implication $(\mathrm{a}) \Rightarrow(\mathrm{b})$ is clear. To prove the converse, assume (b). Then $A$ is a $k[t]$-algebra, and the $H$-comodule structure map

$$
A \rightarrow A \otimes H \cong A \otimes_{k[t]} H[t]
$$

is $k[t]$-linear since $t$ is an $H$-coinvariant element. Thus $B[t] \subset A$ is an $H[t]$-Galois extension, and (a) follows.

(2) Our definition of homotopy equivalence extends the definition introduced in [ $\mathrm{K}$ ] for central Hopf Galois extensions. If $A_{0}$ and $A_{1}$ are right faithfully flat and central $H$-Galois extensions of a commutative $k$-algebra $R$, we can view $A_{0}$ and $A_{1}$ as $R \otimes H$-Galois extensions of the ground ring $R$ as explained above. Then the following are equivalent:

(a) There is a faithfully flat and central $H$-Galois extension $A$ of $R[t]$ with $[i]_{*} A \cong A_{i}$ for $i \in\{0,1\}$.

(b) There is a faithfully flat and central $H$-Galois extension $A$ of $R[t]$ with $R_{[i]} \otimes_{R[t]} A \cong A_{i}$ for $i \in\{0,1\}$.

(c) $A_{0} \sim A_{1}$ as $R \otimes H$-Galois extensions of the ground ring $R$. 
In (b), $R_{[i]}$ denotes $R$ as an $R[t]$-algebra via the $R$-algebra map [i] : $R[t] \rightarrow R$ mapping $t$ onto $i$ for $i \in\{0,1\}$, and $R_{[i]} \otimes_{R[t]} A$ is an algebra by componentwise multiplication.

The equivalence of (a) and (b) follows from the bijectivity of the algebra map $R_{[i]} \otimes_{R[t]} A \rightarrow k_{[i]} \otimes_{k[t]} A$ defined by $r \otimes a \mapsto 1 \otimes r a$, and (b) is equivalent to (c) by Remark 2.4 (1).

(3) Homotopy equivalence is different from isomorphism. See K, Section 4.5] for examples of non-isomorphic homotopy equivalent Hopf Galois extensions.

(4) If the Hopf algebra $H$ is finitely generated and projective as a module over the ground ring $k$, then by a theorem of Kreimer and Takeuchi [KT any $H$-Galois extension $A$ of $B$ is finitely generated projective as a $B$-module. Thus $A$ defines an element $[A]$ in the $K$ theory group $K_{0}(B)$ of $B$. It follows from the definition that, if $A_{0}$ and $A_{1}$ are faithfully flat $H$-Galois extensions of $B$ such that $A_{0} \sim A_{1}$, then there is $\xi \in K_{0}(B[t])$ such that for $i \in\{0,1\}$,

$$
K_{0}([i])(\xi)=\left[A_{i}\right] \in K_{0}(B) .
$$

If $B$ is a regular ring, then by $[\mathrm{B}$, Chapter XII, Theorem 3.1],

$$
K_{0}([0])=K_{0}([1]): K_{0}(B[t]) \rightarrow K_{0}(B) .
$$

(The latter also holds for certain non-regular rings $B$, see $[\mathrm{P}]$.) In this case, $\left[A_{0}\right]=\left[A_{1}\right]$ in $K_{0}(B)$, which means that the element we have constructed in $K_{0}(B)$ is invariant under homotopy equivalence.

If $k$ is a field and the antipode of $H$ is bijective, then any faithfully flat $H$-Galois extension $A$ of $B$ is projective over $B$ by a recent result in Sch, 2.4.9]. Hence the above construction can sometimes be extended to cases when $H$ is no longer finite-dimensional over $k$ (now assumed to be a field). For instance, let $H$ be a cosemisimple Hopf algebra with a decomposition $H \cong \oplus_{i \in I} C_{i}$, where each $C_{i}$ is a finite-dimensional coalgebra over $k$. Then $A \cong \oplus_{i \in I} A \square_{H} C_{i}$. Each summand $A \square_{H} C_{i}$ of $A$ is a finitely generated $B$-module by faithfully flat descent, and it is projective over $B$ since $A$ is $B$-projective. We then obtain a family of elements of $K_{0}(B)$ for which we can argue as above.

Next we show that ()$_{*}$ and ()$^{*}$ induce maps on the homotopy classes.

Proposition 2.5. Let $H$ be a Hopf algebra, and $A_{0}, A_{1}$ right faithfully flat $H$-Galois extensions of $B$ with $A_{0} \sim A_{1}$.

(1) If $\alpha: R \rightarrow S$ is a homomorphism of commutative $k$-algebras, then $\alpha_{*} A_{0} \sim \alpha_{*} A_{1}$.

(2) If $\varphi: K \rightarrow H$ is a homomorphism of Hopf algebras and $K$ is flat over $k$, then $\varphi^{*} A_{0} \sim \varphi^{*} A_{1}$. 
(3) If $\sigma: H \otimes H \rightarrow k$ is an invertible 2-cocycle, then $\left(A_{0}\right)^{\sigma} \sim\left(A_{1}\right)^{\sigma}$.

Proof. Let $A$ be a right faithfully flat $H[t]$-Galois extension of $B[t]$ with $[i]_{*} A \cong A_{i}, i \in\{0,1\}$.

(1) Extend $\alpha$ to the ring homomorphism $\alpha[t]: R[t] \rightarrow S[t]$ by mapping $t$ onto $t$. Then $\alpha[i]=[i] \alpha[t]$. By Proposition $\left[1.2, \alpha[t]_{*} A\right.$ is a right faithfully flat $H[t]$-Galois extension of $B[t]$, and by Proposition 1.4 (1),

$[i]_{*} \alpha[t]_{*} A \cong([i] \alpha[t])_{*} A \cong(\alpha[i])_{*} A \cong \alpha_{*}[i]_{*} A \cong \alpha_{*} A_{i}$ for $i \in\{0,1\}$.

(2) Let $i \in\{0,1\}$. We apply part (3) of Proposition 1.4 to the ring homomorphism $\tau=[i]: k[t] \rightarrow k$ and to the $k[t]$-Hopf algebra map $\varphi[t]: K[t] \rightarrow H[t]$. Then the Hopf algebra map $k_{[i]} \otimes_{k[t]} \varphi$ defined by ground ring extension of $\varphi$ with respect to $[i]$ can be identified with $\varphi$. Hence by Proposition $1.4(3)$,

$$
[i]_{*} \varphi[t]^{*} A \cong \varphi^{*}[i]_{*} A \cong \varphi^{*} A_{i}
$$

(3) By extension $\sigma$ defines an invertible 2-cocycle $\sigma[t]$ of $H[t]$ over the ground ring $k[t]$. By Proposition [1.6] $A^{\sigma[t]}$ is a right faithfully flat $H[t]$-Galois extension of $B[t]$, and

$$
[i]_{*}\left(A^{\sigma[t]}\right) \cong\left([i]_{*} A\right)^{\sigma} \cong\left(A_{i}\right)^{\sigma} \text { for } i \in\{0,1\} .
$$

By Proposition 2.5. $\mathcal{H}_{R \otimes B}(R \otimes H)$ is a covariant functor in commutative $k$-algebras $R$, and $\mathcal{H}_{B}(H)$ is a contravariant functor in $k$-flat Hopf algebras $H$.

We now introduce homotopies between homomorphisms of Hopf algebras.

Definition 2.6. Let $K, H$ be Hopf algebras, and $\varphi: K \rightarrow H$ and $\psi: K \rightarrow H$ Hopf algebra homomorphisms. We say that $\varphi$ and $\psi$ are homotopic $(\varphi \sim \psi)$ if there exists a $k[t]$-Hopf algebra homomorphism $\Phi: K[t] \rightarrow H[t]$ with

$$
[0](\Phi(x))=\varphi(x) \text { and }[1](\Phi(x))=\psi(x)
$$

for all $x \in K$. The map $\Phi$ is called a homotopy between $\varphi$ and $\psi$.

The Hopf algebra map $\varphi: K \rightarrow H$ is a homotopy equivalence if there exists a Hopf algebra homomorphism $\varphi^{\prime}: H \rightarrow K$ with $\varphi \varphi^{\prime} \approx \mathrm{id}_{H}$ and $\varphi^{\prime} \varphi \approx \mathrm{id}_{K}$, where $\approx$ is the equivalence relation generated by $\sim$.

Remarks 2.7. (1) A homotopy $\Phi$ between $\varphi$ and $\psi$ is given by a family $\Phi_{n}: K \rightarrow H(n \geq 0)$ of $k$-linear maps such that for all $x \in K$, $\Phi_{n}(x) \neq 0$ only for finitely many $n$, and for all $x, y \in K$ and all $n \geq 0$,

(a) $\Phi_{n}(x y)=\sum_{i+j=n} \Phi_{i}(x) \Phi_{j}(y)$,

(b) $\Delta\left(\Phi_{n}(x)\right)=\sum_{i+j=n} \Phi_{i}\left(x_{(1)}\right) \otimes \Phi_{j}\left(x_{(2)}\right)$, 
(c) $\Phi_{n}(1)=\delta_{n 0}$,

(d) $\varepsilon\left(\Phi_{n}(x)\right)=\delta_{n 0} \varepsilon(x)$,

(e) $\Phi_{0}=\varphi$

(f) $\sum_{n>0} \Phi_{n}(x)=\psi(x)$.

The homotopy $\Phi$ corresponding to the family $\left(\Phi_{n}\right)$ is defined by

$$
\Phi(x)=\sum_{n \geq 0} \Phi_{n}(x) t^{n}
$$

for all $x \in K$. Note that any family $\left(\Phi_{n}\right)$ of $k$-linear maps with $\Phi_{n}(x) \neq 0$ only for finitely many $n$ and (a)-(d) defines Hopf algebra homomorphisms $\Phi_{0}: K \rightarrow H$ and $\sum_{n \geq 0} \Phi_{n}: K \rightarrow H$.

(2) Let $H=\oplus_{n \geq 0} H(n)$ be an $\mathbb{N}$-graded Hopf algebra. Then the inclusion $H(0) \subset H$ is a homotopy equivalence of Hopf algebras. For the proof let $\iota: H(0) \rightarrow H$ be the inclusion and $\pi: H \rightarrow H(0)$ the projection. Both maps are Hopf algebra homomorphisms, and $\pi \iota=$ $\mathrm{id}_{H(0)}$. We use the same homotopy as before for graded commutative algebras, and define a $k[t]$-Hopf algebra homomorphism

$$
\Phi: H[t] \rightarrow H[t] \text { with } \varphi(x)=x t^{n} \text { for all } x \in H(n), n \geq 0 .
$$

Note that $\Phi$ is a coalgebra map since for all $n \geq 0$,

$$
\Delta(H(n)) \subset \oplus_{i+j=n} H(i) \otimes H(j) \text { and } \varepsilon(H(n))=0 \text { if } n>0 .
$$

Then $\Phi$ is a homotopy between $\iota \pi$ and $\mathrm{id}_{H}$.

The first part of the next theorem generalizes [K, Proposition 2.3].

Theorem 2.8. Let $B$ be an algebra and $H$ a Hopf algebra over $k$.

(1) If $\alpha: R \rightarrow S$ and $\beta: R \rightarrow S$ are homotopic homomorphisms of commutative $k$-algebras, then

$$
\alpha_{*}=\beta_{*}: \mathcal{H}_{R \otimes B}(R \otimes H) \rightarrow \mathcal{H}_{S \otimes B}(S \otimes H) .
$$

(2) If $\varphi: K \rightarrow H$ and $\psi: K \rightarrow H$ are homotopic Hopf algebra homomorphisms, where $K$ is a $k$-flat Hopf algebra, then

$$
\varphi^{*}=\psi^{*}: \mathcal{H}_{B}(H) \rightarrow \mathcal{H}_{B}(K) .
$$

Proof. By Proposition [2.5, all maps are well defined on homotopy classes.

(1) Let $A$ be a right faithfully flat $R \otimes H$-Galois extension of $R \otimes B$. We will show that $\alpha_{*} A \sim \beta_{*} A$.

There is a homotopy $\theta: R \rightarrow S[t]$ with $[0] \theta=\alpha$ and $[1] \theta=\beta$. Then $\theta_{*} A$ is a right faithfully flat $(S \otimes H)[t]$-Galois extension of $(S \otimes B)[t]$, and

$$
[0]_{*} \theta_{*} A \cong([0] \theta)_{*} A \cong \alpha_{*} A \text { and }[1]_{*} \theta_{*} A \cong([1] \theta)_{*} A \cong \beta_{*} A .
$$


(2) Let $A$ be a right faithfully flat $H$-Galois extension of $B$. We will show that $\varphi^{*} A \sim \psi^{*} A$.

There is a homotopy $\Phi: K[t] \rightarrow H[t]$ between $\varphi$ and $\psi$. Define the $H[t]$-Galois extension $A[t]$ of $B[t]$ by ground ring extension via $k \subset k[t]$. Then $\Phi^{*} A[t]$ is a right faithfully flat $K[t]$-Galois extension of $B[t]$ by Proposition [1.3, and for $i \in\{0,1\}$ we have by Proposition 1.4

$$
[i]_{*} \Phi^{*} A[t] \cong\left(k_{[i]} \otimes_{k[t]} \Phi\right)^{*}[i]_{*} A[t] .
$$

Since $A[t]$ is defined via ground ring extension with respect to the inclusion $k \subset k[t]$, we have $[i]_{*} A[t] \cong A$ for $i \in\{0,1\}$. Let us identify the map $k_{[i]} \otimes_{k[t]} \Phi$ defined by ground ring extension via $[i]: k[t] \rightarrow k$. Note that for $i=0,1$,

$$
K \cong k_{[i]} \otimes_{k[t]} K[t], x \mapsto 1 \otimes x
$$

and

$$
k_{[i]} \otimes_{k[t]} H[t] \cong H, 1 \otimes h t^{n} \mapsto h i^{n}
$$

are isomorphisms. The image of an element $x \in K$ under the composition

$$
K \cong k_{[i]} \otimes_{k[t]} K[t] \stackrel{k_{[i]} \otimes \Phi}{\longrightarrow} k_{[i]} \otimes_{k[t]} H[t] \cong H
$$

is $\sum_{n \geq 0} \Phi_{n}(x) i^{n}$, where the homotopy is given by the family $\left(\Phi_{n}\right)_{n \geq 0}$. Hence we can identify $k_{[0]} \otimes_{k[t]} \Phi$ with $\varphi$, and $k_{[1]} \otimes_{k[t]} \Phi$ with $\psi$. This proves our claim that $\varphi^{*} A \sim \psi^{*} A$.

It is clear from Theorem 2.8 that homotopy equivalences induce bijective maps on homotopy classes. Hence Remarks 2.2 (2) and 2.7 (2) imply the following Corollary whose first part generalizes $\mathrm{K}$, Corollary 2.4].

Corollary 2.9. Let $B$ be an algebra and $H$ a Hopf algebra over $k$.

(1) If $R=\oplus_{n \geq 0} R(n)$ is an $\mathbb{N}$-graded commutative algebra with $R(0)=$ $k$, then the inclusion map $\iota: k \rightarrow R$ induces a bijective map

$$
\iota_{*}: \mathcal{H}_{B}(H) \rightarrow \mathcal{H}_{R \otimes B}(R \otimes H) .
$$

(2) If $H=\oplus_{n \geq 0} H(n)$ is an $\mathbb{N}$-graded Hopf algebra with $K=H(0)$, then the inclusion map $\iota: K \rightarrow H$ induces a bijective map

$$
\iota^{*}: \mathcal{H}_{B}(H) \rightarrow \mathcal{H}_{B}(K)
$$

The following examples show that $\mathcal{H}$ cannot be replaced by Gal in Corollary 2.9 (1) and (2). 
Examples 2.10. (1) Let $H$ be a finite-dimensional Hopf algebra over a field $k$, and $R$ a commutative graded $k$-algebra with $R(0)=k$ such that there exists a non-cleft faithfully flat $R \otimes H$-Galois extension of $R$ over the ground ring $R$. Since any $H$-Galois extension of $k$ is cleft, the induced map $\operatorname{Gal}_{k}(H) \rightarrow \operatorname{Gal}_{R}(R \otimes H)$ is not bijective. An example of this type is described in Proposition 3.3 below with $H=k\left[C_{2}\right]$ and $R=k\left[x^{2}, x^{3}\right]$.

(2) We recall Masuoka's computation of $\mathrm{Gal}_{k}(H)$ for the Taft Hopf algebra in [Ma1. Let $k$ be a field, $N>2$ be a natural number, and $q$ a root of unity of order $N$ in $k$. The Taft Hopf algebra of dimension $N^{2}$ is the algebra

$$
H=H_{N^{2}}=k\left\langle g, x \mid g^{N}=1, x^{N}=0, g x g^{-1}=q x\right\rangle
$$

with Hopf algebra structure defined by

$$
\Delta(g)=g \otimes g \text { and } \Delta(x)=1 \otimes x+x \otimes g .
$$

It is an $\mathbb{N}$-graded Hopf algebra with $H(n)=k[G] x^{n}(n \geq 0)$, where $G=G(H)$ is the cyclic group of order $N$ generated by $g$. For any $r \in U(k)$ and $s \in k$ define the $H$-comodule algebra $A_{r, s}$ by

$$
A_{r, s}=k\left\langle a, b \mid a^{N}=r, b^{N}=s, a b a^{-1}=q b\right\rangle
$$

with $H$-comodule algebra structure $\delta$ given by

$$
\delta(a)=a \otimes g \text { and } \delta(b)=1 \otimes x+b \otimes g .
$$

By [Ma1, Proposition 2.17, Lemma 2.19], the map $(r, s) \mapsto A_{r, s}$ defines a bijection

$$
U(k) / U(k)^{N} \times k \cong \operatorname{Gal}_{k}(H) .
$$

On the other hand, for any $r \in U(k)$ define the $k[G]$-comodule algebra $A_{r}$ by

$$
A_{r}=k\left\langle a \mid a^{N}=r\right\rangle \text { with } \delta(a)=a \otimes g .
$$

Then $r \mapsto A_{r}$ defines a bijection

$$
U(k) / U(k)^{N} \cong \operatorname{Gal}_{k}(k[G]) .
$$

If $r \in U(k)$ and $s \in k$, then $A_{r} \rightarrow A_{r, s} \square_{H} k[G], a \mapsto a \otimes g$, is an isomorphism of $k[G]$-comodule algebras. Hence the map $\operatorname{Gal}_{k}(H) \rightarrow$ $\mathrm{Gal}_{k}(k[G])$ induced by the inclusion $k[G]=H(0) \subset H$ can be identified with the projection $U(k) / U(k)^{N} \times k \rightarrow U(k) / U(k)^{N}$. In particular, it is not bijective.

Without using the explicit computation of $\mathrm{Gal}_{k}(H)$, we know from Corollary 2.9 (2) that the inclusion $k[G] \subset H$ defines a bijection

$$
\mathcal{H}_{k}(H) \cong \mathcal{H}_{k}(k[G]) \text {. }
$$


Since $G$ is cyclic of order $N$,

$$
\mathcal{H}_{k}(k[G]) \cong \mathrm{Gal}_{k}(k[G]) \cong H^{2}(G, U(k)) \cong U(k) / U(k)^{N}
$$

by Proposition 3.2 (1) below.

For later use we note the following combination of our results on twisted and graded Hopf algebras.

Corollary 2.11. Let $B$ be an algebra, $H=\oplus_{n \geq 0} H(n)$ an $\mathbb{N}$-graded Hopf algebra, and $K=H(0)$. Let $\sigma: H \times H \rightarrow k$ be an invertible 2-cocycle such that $\sigma(x, y)=\varepsilon(x) \varepsilon(y)$ for all $x, y \in K$. Then $K$ is a Hopf subalgebra of $H^{\sigma}$ and the inclusion $\iota: K \rightarrow H^{\sigma}$ induces a bijective map $\iota^{*}: \mathcal{H}_{B}\left(H^{\sigma}\right) \rightarrow \mathcal{H}_{B}(K)$.

Proof. Let $A$ be a faithfully flat $H$-Galois extension of $B$. By Proposition [1.6] and Corollary 2.9 (2), it is enough to show that

$$
A \square_{H} K \cong A^{\sigma} \square_{H^{\sigma}} K
$$

as $K$-Galois extensions of $B$. Since $A \square_{H} K=A^{\sigma} \square_{H^{\sigma}} K$ as $K$-comodules, it suffices to check that the algebra structures of $A \square_{H} K$ and $A^{\sigma} \square_{H^{\sigma}} K$ coincide. Let $\sum_{i} a_{i} \otimes x_{i}$ and $\sum_{j} b_{j} \otimes y_{j}$ be elements of $A \square_{H} K$. We denote the multiplication in $A^{\sigma} \square_{H^{\sigma}} K$ by ${ }_{\sigma}$. Then it follows from the definition of the cotensor product that

$$
\begin{aligned}
\left(\sum_{i} a_{i} \otimes x_{i}\right) \cdot_{\sigma}\left(\sum_{j} b_{j} \otimes y_{j}\right) & =\sum_{i, j} a_{i} \cdot_{\sigma} b_{j} \otimes x_{i} y_{j} \\
& =\sum_{i, j} a_{i(0)} b_{j(0)} \sigma^{-1}\left(a_{i(1)}, b_{j(1)}\right) \otimes x_{i} y_{j} \\
& =\sum_{i, j} a_{i} b_{j} \sigma^{-1}\left(x_{i(1)}, y_{j(1)}\right) \otimes x_{i(2)} y_{j(2)} \\
& =\left(\sum_{i} a_{i} \otimes x_{i}\right)\left(\sum_{j} b_{j} \otimes y_{j}\right)
\end{aligned}
$$

since $\sigma^{-1}\left(x_{i(1)}, y_{j(1)}\right)=\varepsilon\left(x_{i(1)}\right) \varepsilon\left(y_{j(1)}\right)$.

\section{Homotopy of ABELIAN GROUP FUnCTORS, AND EXAMPlES FROM GROUP ALGEBRAS}

In [Sw1, Section 4] Swan constructed a homotopy functor out of any functor defined on a category of commutative algebras. Our definition of $\mathcal{H}_{B}(H)$ is a special case of this construction.

A functor $F$ from the category of commutative $k$-algebras to the category of sets is called a homotopy functor if for any commutative 
$k$-algebra $R$,

$$
F(R[0])=F(R[1]): F(R[t]) \rightarrow F(R)
$$

or, equivalently, if the inclusion $\iota: R \subset R[t]$ induces a bijection $F(R) \cong$ $F(R[t])$.

For any commutative $k$-algebra $R$ let $\bar{F}(R)$ be the coequalizer of the $\operatorname{maps}[1]_{*}=F(R[1]),[0]_{*}=F(R[0]): F(R[t]) \rightarrow F(R)$.

In general, if $M, N$ are sets and $f, g: M \rightarrow N$ are maps, the coequalizer of the pair $f, g$ is described as follows. For all $x, y \in N$, define $x \sim y$ if there is an element $z \in M$ with $f(z)=x$ and $g(z)=y$. Then the coequalizer of the pair $f, g$ is the quotient map $N \rightarrow N / \approx$, where $\approx$ is the equivalence relation generated by $\sim$.

Let us say that two elements $x, y \in F(R)$ are homotopy equivalent if $x \approx y$.

From the definition there is a natural transformation $\eta: F \rightarrow \bar{F}$. By [Sw1, Lemma 4.2], $\eta$ is universal for maps of $F$ into homotopy functors. Thus $\bar{F}$ is the largest quotient of $F$ which is a homotopy functor.

In particular, if $F$ is the functor

$$
R \mapsto F(R)=\operatorname{Gal}_{R \otimes B}(R \otimes H),
$$

then $\bar{F}(R)=\mathcal{H}_{R \otimes B}(R \otimes H)$.

Remark 3.1. Let $F$ be a functor from commutative $k$-algebras to abelian groups. Let $\bar{F}$ be the largest quotient of the underlying set functor of $F$ which is a homotopy functor. Then for any commutative algebra $R$, the relation $\sim$ coincides with $\approx$ on $F(R)$, and $\bar{F}(R)$ is the cokernel of $[1]_{*}-[0]_{*}$.

Indeed, using standard notation (see $\mathrm{B}$, Chapter XII]), we denote by $N F(R)$ the kernel of the map $[0]_{*}: F(R[t]) \rightarrow F(R)$. It splits off $F(R[t])$ and we have the following functorial decomposition: $F(R[t])=$ $F(R) \oplus N F(R)$. It is immediate to see that for two elements $x, y \in$ $F(R)$ we have $x \sim y$ if and only if $x-y$ belongs to the image of the map

$$
[1]_{*}: N F(R) \rightarrow F(R[t]) \rightarrow F(R),
$$

which is equal to the image of $[1]_{*}-[0]_{*}: F(R[t]) \rightarrow F(R)$. Therefore,

$$
\bar{F}(R) \cong F(R) /[1]_{*} N F(R) .
$$

Let $H$ be a cocommutative $k$-flat Hopf algebra. Then the set $\operatorname{Gal}_{k}(H)$ of isomorphism classes of Galois objects of $H$ form an abelian group (see for example [C, 10.5.3]). If $A$ and $A^{\prime}$ are $H$-Galois objects, then their product in $\operatorname{Gal}_{k}(H)$ is the isomorphism class of $A \square_{H} A^{\prime}$, where $A^{\prime}$ is viewed as a left $H$-comodule algebra, which is possible since $H$ 
is cocommutative. In a very special case this group structure already appeared in [Ha]. Thus

$$
R \mapsto \operatorname{Gal}_{R}(R \otimes H)
$$

is a functor from commutative $k$-algebras to abelian groups, and $\mathcal{H}_{k}(H)$ is the cokernel of the homorphism

$$
[1]_{*}-[0]_{*}: \operatorname{Gal}_{k[t]}(k[t] \otimes H) \rightarrow \operatorname{Gal}_{k}(H) .
$$

We are interested in the case when $H=R[G]$ is the group algebra of a group $G$ over a commutative ring $R$. Then Galois objects are $G$ strongly graded algebras, i.e., we have $A=\oplus_{g \in G} A_{g}, A_{1}=R$, and for all $g, h \in G$, the multiplication map

$$
A_{g} \otimes_{R} A_{h} \rightarrow A_{g} A_{h}=A_{g h}
$$

is bijective (see [M, 8.1.7]). Thus

$$
\Phi(A): G \rightarrow \operatorname{Pic}(R), g \mapsto\left[A_{g}\right],
$$

is a group homomorphism. Here $\operatorname{Pic}(R)$ is the abelian group of isomorphism classes of invertible $R$-modules.

Suppose that for all $g \in G, A_{g} \cong R$ as an $R$-module. Then any $A_{g}$ contains an invertible element $u_{g}$ of $A$, and $A$ is $R[G]$-cleft with the $R[G]$-colinear and invertible map $R[G] \rightarrow A$ defined by $g \mapsto u_{g}$.

Let $\sigma: G \times G \rightarrow U(R)$ be a 2-cocycle of the group $G$ acting trivially on $U(R)$. The twisted group algebra $\Psi(\sigma)=R_{\sigma}[G]$ is a cleft $R[G]$ Galois object [M, 7.1.5]. Recall that $R_{\sigma}[G]=R[G]$ as a coalgebra with twisted multiplication $g \cdot{ }_{\sigma} h=\sigma(g, h) g h$ on basis elements $g, h \in G$. The maps $\Phi$ and $\Psi$ define an exact sequence of abelian groups

$$
0 \rightarrow H^{2}(G, U(R)) \stackrel{\Psi}{\rightarrow} \operatorname{Gal}_{R}(R[G]) \stackrel{\Phi}{\rightarrow} \operatorname{Hom}(G, \operatorname{Pic}(R)) .
$$

Both homomorphisms $\Phi$ and $\Psi$ are natural transformations of abelian group functors on the category of commutative rings $R$.

Let $\overline{\operatorname{Hom}}(G, \operatorname{Pic}(R))$ be the cokernel of

$$
\operatorname{Hom}\left(G,[1]_{*}-[0]_{*}\right): \operatorname{Hom}(G, \operatorname{Pic}(R[t])) \rightarrow \operatorname{Hom}(G, \operatorname{Pic}(R)) .
$$

Proposition 3.2. Let $G$ be a group and $R$ a reduced commutative ring.

(1) If $\operatorname{Pic}(R[t])=0$, then

$$
H^{2}(G, U(R)) \cong \operatorname{Gal}_{R}(R[G])=\mathcal{H}_{R}(R[G]) .
$$

(2) If $G$ is a finite abelian group, then $\Phi$ and $\Psi$ define an exact sequence

$$
H^{2}(G, U(R)) \rightarrow \mathcal{H}_{R}(R[G]) \rightarrow \overline{\operatorname{Hom}}(G, \operatorname{Pic}(R)) \rightarrow 0 .
$$


Proof. (1) The first isomorphism follows from (3.1) since $\operatorname{Pic}(R)=0$ as a direct summand in $\operatorname{Pic}(R[t])=0$. It remains to show that homotopic $R[G]$-Galois objects $A_{0}$ and $A_{1}$ are isomorphic. By assumption there is an $R[t][G]$-Galois object $A$ with $[i]_{*} A \cong A_{i}, i=0,1$. Since $\operatorname{Pic}(R[t])=$ 0 , there is a 2-cocycle $\sigma: G \times G \rightarrow U(R[t])$ with $A \cong R[t]_{\sigma}[G]$. The ring $R$ being reduced, we have $U(R[t])=U(R)$, and $\sigma$ takes values in $U(R)$. Hence,

$$
[i]_{*} A \cong[i]_{*} R[t]_{\sigma}[G] \cong R_{\sigma}[G]
$$

for $i=0,1$.

(2) Define group homomorphisms $f_{1}, f_{2}, f_{3}$ by

$$
\begin{gathered}
f_{1}=[1]_{*}-[0]_{*}: H^{2}(G, U(R[t])) \rightarrow H^{2}(G, U(R)), \\
f_{2}=[1]_{*}-[0]_{*}: \operatorname{Gal}_{R[t]}(R[t][G]) \rightarrow \operatorname{Gal}_{R}(R[G]), \text { and } \\
f_{3}=\operatorname{Hom}\left(G,[1]_{*}-[0]_{*}\right): \operatorname{Hom}(G, \operatorname{Pic}(R[t])) \rightarrow \operatorname{Hom}(G, R) .
\end{gathered}
$$

It is known (see [C, 10.7.1]) that the map $\Phi$ in (3.1) is an epimorphism for any commutative ring $R$ if the group $G$ is finite and abelian. Then $\left(f_{1}, f_{2}, f_{3}\right)$ define a homomorphism of short exact sequences (3.1) for $R[t]$ and for $R$, and we have an exact sequence

$$
\operatorname{Coker}\left(f_{1}\right) \rightarrow \operatorname{Coker}\left(f_{2}\right) \rightarrow \operatorname{Coker}\left(f_{3}\right) \rightarrow 0 .
$$

Since $R$ is reduced, $U(R)=U(R[t])$. Therefore

$$
[0]_{*}=[1]_{*}: U(R[t]) \rightarrow U(R),
$$

hence $f_{1}=0$, and $\operatorname{Coker}\left(f_{1}\right)=H^{2}(G, U(R))$. By Remark 3.1. Coker $\left(f_{2}\right)=$ $\mathcal{H}_{R}(R[G])$, and $\operatorname{Coker}\left(f_{3}\right)=\overline{\operatorname{Hom}}(G, \operatorname{Pic}(R))$.

Suppose in the situation of Proposition 3.2 (2) that

$$
[1]_{*}-[0]_{*}: \operatorname{Pic}(R[t]) \rightarrow \operatorname{Pic}(R)
$$

is a split epimorphism. Then $\Psi$ induces an epimorphism

$$
\left.H^{2}(G, U(R)) \rightarrow \operatorname{Gal}_{R}(R[G]]\right) \rightarrow \mathcal{H}_{R}(R[G]) .
$$

Hence, if there is a non-cleft $R[G]$-Galois object, then there is a noncleft $R[G]$-Galois object which is homotopically trivial, that is, homotopically equivalent to the group algebra. We will now explicitly construct such an example. 
Let $k$ be a field, $R$ a commutative $k$-algebra without zero divisors, and $F$ the field of fractions of $R$. If $P$ is a fractional ideal of $R$ (i.e., an $R$-submodule of $F$ ) such that $P^{2}=R$, then we can consider the $C_{2}$-graded $R$-submodule $R * P$ of $F\left[C_{2}\right]$ defined by

$$
(R * P)_{0}=R \text { and }(R * P)_{1}=P g,
$$

where $g$ generates the group $C_{2}$. The condition $P^{2}=R$ implies that $R * P$ is a strongly $C_{2}$-graded subalgebra of $F\left[C_{2}\right]$. It follows that $R * P$ is a $k\left[C_{2}\right]$-Hopf Galois extension with coinvariants $R$. The extension $R * P$ is cleft if and only if $P$ is free.

We now assume that $k$ is of characteristic 2 and $R$ is not seminormal, i.e., there is an element $a \in F \backslash R$ such that $a^{2}$ and $a^{3}$ belong to $R$. We also assume that any unit $u$ of the subring of $F$ generated by $R$ and $a$ such that $u(1+a) \in R$ belongs to $R$. As an example of such a ring, take $R$ to be the subring $k\left[x^{2}, x^{3}\right]$ in the polynomial algebra $k[x]$, and $a=x$.

Following [Sw2, Section 7] and L, Section 2B, Example 2.15], we consider the Schanuel fractional ideal $P_{a}=\left(1+a, a^{2}\right)$ of $R$ generated by $1+a$ and $a^{2}$ in $F$. It follows from Equation $(2.15 \mathrm{~A})$ of $[\mathrm{L}$ and the restriction we put on the characteristic of $k$ that $P_{a}^{2}=R$. By the above we obtain a $k\left[C_{2}\right]$-Hopf Galois extension $R * P_{a}$. We claim the following.

Proposition 3.3. The $k\left[C_{2}\right]$-Hopf Galois extension $R * P_{a}$ is not cleft, but it is homotopically trivial.

Proof. (a) By $\left[\mathrm{L}\right.$, Proposition 2.15C], the $R$-module $P_{a}$ is not free. Therefore $R * P_{a}$ is not cleft.

(b) In the polynomial ring $R[t]$ the element at of $F[t]$ is not in $R[t]$, but $a^{2} t^{2}$ and $a^{3} t^{3}$ belong to $R[t]$. We can then consider the Schanuel fractional ideal $P_{a t}$ of $R[t]$ generated by $1+a t$ and $a^{2} t^{2}$ in the field of fractions of $R[t]$. This leads to the non-cleft $k\left[C_{2}\right]$-Hopf Galois extension $R[t] * P_{a t}$ whose coinvariants are $R[t]$.

We claim that $R[t] * P_{a t} /(t-1) \cong R * P_{a}$ and $R[t] * P_{a t} /(t) \cong R\left[C_{2}\right]$. This will prove that the Galois extension $R * P_{a}$ is homotopy equivalent to the trivial extension $R\left[C_{2}\right]$. To prove the claim, it suffices to check that we have the following isomorphisms of $R$-modules:

$$
P_{a t} /(t-1) P_{a t} \cong P_{a} \text { and } P_{a t} / t P_{a t} \cong R \text {. }
$$

According to [Sw2, Proof of Theorem 7.1], the $R[t]$-module $P_{a t}$ is the image of the idempotent matrix

$$
M_{t}=\left(\begin{array}{cc}
1-a^{4} t^{4} & a^{2} t^{2}+a^{3} t^{3} \\
\left(1+a^{2} t^{2}\right)\left(a^{2} t^{2}-a^{3} t^{3}\right) & a^{4} t^{4}
\end{array}\right) .
$$


Therefore, $P_{a t} /(t-1) P_{a t}$ is the image of the idempotent

$$
M_{1}=\left(\begin{array}{cc}
1-a^{4} & a^{2}+a^{3} \\
\left(1+a^{2}\right)\left(a^{2}-a^{3}\right) & a^{4}
\end{array}\right),
$$

which is the $R$-module $P_{a}$, and $P_{a t} / t P_{a t}$ is the image of the idempotent $M_{0}=\left(\begin{array}{ll}1 & 0 \\ 0 & 0\end{array}\right)$, which is a free $R$-module of rank 1 .

A fractional ideal as above is an invertible (rank one) projective $R$ module. Hence it represents an element $[P]$ of order 2 in the Picard group $\operatorname{Pic}(R)$ of $R$. As observed above, the Hopf-Galois extension $R * P$ is cleft if and only if $[P]=0$ in $\operatorname{Pic}(R)$.

We have seen in Remark 3.1 that homotopy equivalence in $\operatorname{Pic}(R)$ is different from equality provided $[1]_{*} N \operatorname{Pic}(R)$ is non-zero. A necessary condition for the non-vanishing of $[1]_{*} N \operatorname{Pic}(R)$ is the non-vanishing of $N \operatorname{Pic}(R)$, which by [Sw2, Theorem 1] is equivalent to the reduced quotient $R_{\text {red }}$ of $R$ not being seminormal.

The construction of the Schanuel module $P_{a t}$ leading to the example of the Proposition above, together with [L, (2.23B) and Theorem 2.23], shows there exist injective maps

$$
\iota: R / J \rightarrow \operatorname{Pic}(R) \text { and } \iota_{t}: R / J \rightarrow N \operatorname{Pic}(R)
$$

respectively given by

$$
\iota(\bar{r})=\left[\left(1+r a, a^{2}\right)\right] \text { and } \iota_{t}(\bar{r})=\left[\left(1+r a t, a^{2} t^{2}\right)\right],
$$

where $J=\{b \in R \mid a b \in R\}$ is the so-called conductor. Moreover, we have $\iota=[1]_{*} \circ \iota_{t}$. This implies that $\operatorname{Pic}(R) /[1]_{*} N \operatorname{Pic}(R)$ is a quotient of the cokernel $\operatorname{Pic}(R) /(R / J)$ of $\iota$, thus providing an "upper bound" to the set of homotopy classes in $\operatorname{Pic}(R)$.

Example 3.4. If $R=k\left[x^{2}, x^{3}\right]$, then $\operatorname{Pic}(R) \cong R / J \cong k$, and the composition $\iota$

$$
R / J \stackrel{\iota t}{\rightarrow} N \operatorname{Pic}(R) \subset \operatorname{Pic}(R[t]) \stackrel{[1]_{*}-[0]_{*}}{\longrightarrow} \operatorname{Pic}(R)
$$

is an isomorphism $[\mathrm{L},(2.23 \mathrm{C})]$. Hence $[1]_{*}-[0]_{*}: \operatorname{Pic}(R[t]) \rightarrow \operatorname{Pic}(R)$ is a split epimorphism. In particular, all elements of the Picard group are homotopy equivalent to 0 .

\section{EXAMPLES FROM QUANTUM GROUPS}

From now on we assume that the ground ring $k$ is a field. In this Section we apply our previous results to the Drinfeld-Jimbo quantum groups $U_{q}(\mathfrak{g})$ and related finite-dimensional Hopf algebras. It turns out 
that the computation of the homotopy classes for these Hopf algebras can be reduced to the case of abelian group algebras.

The general idea is to present these Hopf algebras as 2-cocycle twists of graded Hopf algebras. Such a presentation was given for finitedimensional quantum groups in [AS], Di]. We adapt this approach to deal with $U_{q}(\mathfrak{g})$ and prove some slightly more general results which may be of independent interest.

We begin with the definition of the generalized quantum double via twisting following [DT].

Let $U, A$ be Hopf algebras, and $\tau: U \otimes A \rightarrow k$ a skew-pairing, that is a linear map such that for all $u, v \in U$ and $a, b \in A$,

$$
\begin{aligned}
\tau(u v, a) & =\tau\left(u, a_{(1)}\right) \tau\left(v, a_{(2)}\right), \\
\tau(u, a b) & =\tau\left(u_{(2)}, a\right) \tau\left(u_{(1)}, b\right), \\
\tau(u, 1) & =\varepsilon(u), \tau(1, a)=\varepsilon(a) .
\end{aligned}
$$

We assume that the antipode of $A$ is invertible. Then $\tau$ is invertible with respect to convolution, and its inverse $\tau^{-1}$ is given for all $u \in U$, $a \in A$ by

$$
\tau^{-1}(u, a)=\tau(S(u), a)=\tau\left(u, S^{-1}(a)\right) .
$$

Skew-pairings can be equivalently described as Hopf algebra homomorphisms $\varphi: U \rightarrow\left(A^{0}\right)^{\text {cop }}$, where $A^{0}$ is the dual Hopf algebra (see [M], Section 9.1]), and for any Hopf algebra $H$ we denote by $H^{\text {cop }}$ the algebra $H$ with the opposite comultiplication. The connection between $\tau$ and $\varphi$ is given by

$$
\tau(u, a)=\varphi(u)(a)
$$

for all $u \in U$ and $a \in A$. We define the associated 2-cocycle $\sigma_{\tau}=\sigma$ on the tensor product Hopf algebra $U \otimes A$ by

$$
\sigma(u \otimes a, v \otimes b)=\varepsilon(u) \tau(v, a) \varepsilon(b)
$$

for all $u, v \in U$ and $a, b \in A$. By definition, $(U \otimes A)^{\sigma}$ is the generalized quantum double of $U, A$ and $\tau$.

The multiplication ${ }_{\sigma}$ in $(U \otimes A)^{\sigma}$ is given by

$$
(u \otimes a) \cdot_{\sigma}(v \otimes b)=u \tau\left(v_{(1)}, a_{(1)}\right) v_{(2)} \otimes a_{(2)} \tau^{-1}\left(v_{(3)}, a_{(3)}\right) b
$$

for all $u, v \in U$ and $a, b \in A$.

We now generalize some results on twisting from Didt's thesis Di.

We consider another skew-pairing $\widetilde{\tau}$ with associated 2-cocycle $\widetilde{\sigma}$. Then $\rho=\widetilde{\sigma} \sigma^{-1}:(U \otimes A)^{\sigma} \otimes(U \otimes A)^{\sigma} \rightarrow k$ is a 2-cocycle on the twisted Hopf algebra $(U \otimes A)^{\sigma}$ associated to the skew-pairing $\widetilde{\tau} \tau^{-1}$, and

$$
(U \otimes A)^{\widetilde{\sigma}}=\left((U \otimes A)^{\sigma}\right)^{\rho} .
$$


Lemma 4.1. Let $y \in G(U), g \in G(A)$. Then the following are equivalent:

(1) For all $a \in A, u \in U$,

$$
\tau(y, a)=\widetilde{\tau}(y, a) \text { and } \tau(u, g)=\widetilde{\tau}(u, g) .
$$

(2) For all $a \in A, u \in U$,

$$
\rho(u \otimes a, y \otimes g)=\varepsilon(u) \varepsilon(a)=\rho(y \otimes g, u \otimes a) .
$$

Proof. This is easily checked since for all $a \in A, u \in U$,

$$
\rho(u \otimes a, y \otimes g)=\varepsilon(u) \widetilde{\tau}\left(y, a_{(1)}\right) \tau^{-1}\left(y, a_{(2)}\right),
$$

and

$$
\rho(y \otimes g, u \otimes a)=\widetilde{\tau}\left(u_{(1)}, g\right) \tau^{-1}\left(u_{(2)}, g\right) \varepsilon(a) .
$$

We will use the previous Lemma to define a 2-cocycle on a quotient Hopf algebra modulo a central subgroup algebra.

Lemma 4.2. Let $H$ be a Hopf algebra, and $\rho: H \otimes H \rightarrow k$ an invertible 2-cocycle. Let $G$ be a subgroup of $G(H)$, and assume that $G$ is central in $H$ and that $\rho(g, x)=\varepsilon(x)=\rho(x, g)$ for all $g \in G$ and $x \in H$. Then $G$ is central in $H^{\rho}$, and $\rho$ induces an invertible 2 -cocycle $\bar{\rho}$ of the quotient Hopf algebra $H /(k[G])^{+} H$ such that

$$
\left(H /(k[G])^{+} H\right)^{\bar{\rho}}=H^{\rho} /(k[G])^{+} H^{\rho} .
$$

Proof. In order to see that the map

$$
\bar{\rho}: H /(k[G])^{+} H \otimes H /(k[G])^{+} H \rightarrow k, \bar{x} \otimes \bar{y} \mapsto \rho(x, y),
$$

is well defined, we have to show that

$$
\rho(g x, y)=\rho(x, y)=\rho(x, y g)
$$

for all $x, y \in H, g \in G$. This follows from the assumption and the two cases of the 2-cocycle condition (1.1) when $x=g$ and $z=g$. The rest of the Lemma is then obvious since $(k[G])^{+} H=(k[G])^{+} H^{\rho}$ by (1.2).

In the sequel we will assume that the group $G(A)$ is abelian and that there exist elements $a_{1}, \ldots, a_{t} \in A \backslash 0, g_{1}, \ldots, g_{t} \in G(A)$, and $\chi_{1}, \ldots, \chi_{t} \in \operatorname{Hom}(G(A), U(k))$ such that $A$ as an algebra is generated by $G(A)$ and by $a_{1}, \ldots, a_{t}$, and for all $j$,

$$
\begin{array}{r}
\Delta\left(a_{j}\right)=g_{j} \otimes a_{j}+a_{j} \otimes 1, \\
g a_{j} g^{-1}=\chi_{j}(g) a_{j} \text { for all } g \in G(A), \\
\chi_{j}\left(g_{j}\right) \neq 1 .
\end{array}
$$


Similarly, we assume that the group $G(U)$ is abelian and that there exist elements $u_{1}, \ldots, u_{s} \in U \backslash 0, y_{1}, \ldots, y_{s} \in G(U)$, and $\eta_{1}, \ldots, \eta_{s} \in$ $\operatorname{Hom}(G(U), U(k))$ such that $U$ as an algebra is generated by $G(U)$ and by $u_{1}, \ldots, u_{s}$, and for all $i$,

$$
\begin{array}{r}
\Delta\left(u_{i}\right)=y_{i} \otimes u_{i}+u_{i} \otimes 1, \\
y u_{i} y^{-1}=\eta_{i}(y) u_{i} \text { for all } y \in G(U), \\
\eta_{i}\left(y_{i}\right) \neq 1 .
\end{array}
$$

Our assumptions for $U$ (and similarly for $A$ ) imply for all $i$

$$
S\left(y_{i}\right)=y_{i}^{-1}, \varepsilon\left(y_{i}\right)=1, S\left(u_{i}\right)=-y_{i}^{-1} u_{i}, \varepsilon\left(u_{i}\right)=0 .
$$

Note that, if $\tau: U \otimes A \rightarrow k$ is a skew-pairing, then

$$
\tau\left(y, a_{j}\right)=0 \text { for all } y \in G(U), 1 \leq j \leq t,
$$

since by (4.2) the map $\gamma: A \rightarrow k, a \mapsto \tau(y, a)$, is an algebra map, and for any algebra map $\gamma, \gamma\left(a_{j}\right)=0$ since $\chi_{j}\left(g_{j}\right) \neq 1$ by (4.9), and by (4.8), $\gamma\left(a_{j}\right)=\gamma\left(g_{j} a_{j} g_{j}^{-1}\right)=\chi_{j}\left(g_{j}\right) \gamma\left(a_{j}\right)$.

In the same way,

$$
\tau\left(u_{i}, g\right)=0 \text { for all } g \in G(A), 1 \leq i \leq s .
$$

Generalizing an argument in the proof of [AS, Theorem 5.17] on page 17, we describe central group-like elements in $(U \otimes A)^{\sigma}$.

Lemma 4.3. Let $y \in G(U), g \in G(A)$. Then the following are equivalent:

(1) The group-like element $y \otimes g^{-1}$ is central in $(U \otimes A)^{\sigma}$.

(2) For all $1 \leq i \leq s, 1 \leq j \leq t$,

$$
\eta_{i}(y)=\tau\left(y_{i}, g\right) \text { and } \chi_{j}\left(g^{-1}\right)=\tau\left(y, g_{j}\right) \text {. }
$$

Proof. Since for all $u \in U, a \in A, u \otimes a=(u \otimes 1) \cdot{ }_{\sigma}(1 \otimes a)$, the element $y \otimes g^{-1}$ is central if it commutes with all $u^{\prime} \otimes 1$, where $u^{\prime}$ is group-like or $u^{\prime}=u_{i}$ for some $i$, and with all $1 \otimes a^{\prime}$, where $a^{\prime}$ is group-like or $a^{\prime}=a_{j}$ for some $j$.

Since the groups $G(U)$ and $G(A)$ are abelian, $y \otimes g^{-1}$ commutes with all $u^{\prime} \otimes a^{\prime}$, where $u^{\prime}$ and $a^{\prime}$ are group-like.

Using (4.10), we compute

$$
\begin{aligned}
\left(y \otimes g^{-1}\right) \cdot_{\sigma}\left(u_{i} \otimes 1\right)= & y \tau\left(u_{i(1)}, g^{-1}\right) u_{i(2)} \otimes g^{-1} \tau^{-1}\left(u_{i(3)}, g^{-1}\right) \\
= & y \tau\left(y_{i}, g^{-1}\right) y_{i} \otimes g^{-1} \tau^{-1}\left(u_{i}, g^{-1}\right) \\
& +y \tau\left(y_{i}, g^{-1}\right) u_{i} \otimes g^{-1} \tau^{-1}\left(1, g^{-1}\right) \\
& +y \tau\left(u_{i}, g^{-1}\right) \otimes g^{-1} \tau^{-1}\left(1, g^{-1}\right) \\
= & y \tau\left(y_{i}, g^{-1}\right) u_{i} \otimes g^{-1}
\end{aligned}
$$


since the first and last summand vanish by (4.14). Since

$$
\left(u_{i} \otimes 1\right) \cdot_{\sigma}\left(y \otimes g^{-1}\right)=u_{i} y \otimes g^{-1},
$$

it follows from (4.11) that $y \otimes g^{-1}$ commutes with $u_{i} \otimes 1$ if and only if $\eta_{i}(y)=\tau\left(y_{i}, g\right)$. Similarly, $y \otimes g$ commutes with $1 \otimes a_{j}$ if and only $\chi_{j}\left(g^{-1}\right)=\tau\left(y, g_{j}\right)$.

The next Proposition is an immediate consequence of the previous Lemmas 4.1, 4.2, 4.3, together with (4.13) and (4.14). It is formulated in [Di] in a special case for the Hopf algebras studied in [AS].

Proposition 4.4. Let $U, A$ be Hopf algebras as above and

$$
\tau, \widetilde{\tau}: U \otimes A \rightarrow k
$$

invertible skew-pairings with associated 2-cocycles $\sigma, \widetilde{\sigma}$. Assume that $s=t$, and

$$
\begin{array}{r}
\tau\left(y_{i}, g\right)=\widetilde{\tau}\left(y_{i}, g\right), \tau\left(y, g_{i}\right)=\widetilde{\tau}\left(y, g_{i}\right), \\
\tau\left(y_{i}, g_{j}\right)=\chi_{j}\left(g_{i}^{-1}\right)=\eta_{i}\left(y_{j}\right),
\end{array}
$$

for all $y \in G(U), g \in G(A), 1 \leq i, j \leq t$.

Then the subgroup $G$ generated by all $y_{i} \otimes g_{i}^{-1}, 1 \leq i \leq t$, is central in the twisted Hopf algebras $H=(U \otimes A)^{\sigma}$ and $\widetilde{H}=(U \otimes A)^{\widetilde{\sigma}}$, and the quotient Hopf algebra $\widetilde{H} /(k[G])^{+} \widetilde{H}$ is a 2-cocycle-twist of $H /(k[G])^{+} H$.

In the situation of Proposition 4.4 we will denote the images of

$$
u_{i} \otimes 1, y_{i} \otimes 1,1 \otimes a_{j}, 1 \otimes g_{j}, 1 \leq i, j \leq t, \text { in } H=(U \otimes A)^{\sigma}
$$

respectively by $u_{i}, y_{i}, a_{j}, g_{j}$. Then a somewhat lenghty calculation using the multiplication rule (4.6) together with (4.7), (4.10), (4.13), (4.14) and the equality

$$
\tau^{-1}\left(u_{j}, a_{i}\right)=\tau\left(S\left(u_{j}\right), a_{i}\right)=-\tau\left(y_{j}^{-1}, g_{i}\right) \tau\left(u_{j}, a_{i}\right)
$$

shows that for all $1 \leq i, j \leq t$,

$$
a_{i} u_{j}-\eta_{j}\left(y_{i}\right) u_{j} a_{i}=\tau\left(u_{j}, a_{i}\right)\left(1-y_{j} g_{i}\right) .
$$

By another abuse of language we use the same symbols $u_{i}, y_{i}, a_{j}, g_{j}$ for the images of these elements in the quotient Hopf algebra $H /(k[G])^{+} H$. Then $y_{j}=g_{j}$ for all $j$ in $H /(k[G])^{+} H$, and by multiplying with $g_{j}^{-1}$ we obtain from (4.17)

$$
a_{i} u_{j}^{\prime}-u_{j}^{\prime} a_{i}=\tau\left(u_{j}, a_{i}\right)\left(g_{j}^{-1}-g_{i}\right),
$$

with $u_{j}^{\prime}=u_{j} g_{j}^{-1}$. 
Let us now look at the concrete example of the Drinfeld-Jimbo algebras $U_{q}(\mathfrak{g})$ (see [J], Chapter 4]). Let $k$ be a field of characteristic $\neq 2,3$. Let $\left(a_{i j}\right)_{1 \leq i, j \leq t}$ be a Cartan matrix of finite type of a semisimple complex Lie algebra $\mathfrak{g}$, and for all $1 \leq i \leq t$ let $d_{i} \in\{1,2,3\}$ with $d_{i} a_{i j}=d_{j} a_{j i}$ for all $i, j$. Let $0 \neq q \in k$ and $q_{i}=q^{d_{i}}$ for all $i$. Assume that $q^{2 d_{i}} \neq 1$ for all $i$.

Let $A=U_{q}^{\geq 0}(\mathfrak{g})$ be the algebra with generators $E_{i}, K_{i}, K_{i}^{-1}, 1 \leq$ $i \leq t$ and relations

$$
\begin{gathered}
K_{i} K_{j}=K_{j} K_{i}, K_{i} K_{i}^{-1}=1=K_{i}^{-1} K_{i} \text { for all } i, j, \\
K_{i} E_{j} K_{i}^{-1}=q^{d_{i} a_{i j}} E_{j} \text { for all } i, j, \\
\sum_{r=0}^{1-a_{i j}}(-1)^{r}\left[\begin{array}{c}
1-a_{i j} \\
r
\end{array}\right]_{q_{i}} E_{i}^{1-a_{i j}-r} E_{j} E_{i}^{r}=0 \text { for all } i \neq j .
\end{gathered}
$$

The algebra $A$ is a Hopf algebra with comultiplication

$$
\Delta\left(K_{i}\right)=K_{i} \otimes K_{i}, \Delta\left(E_{i}\right)=K_{i} \otimes E_{i}+E_{i} \otimes 1 \text { for all } i .
$$

Let $U=U_{q}^{\leq 0}(\mathfrak{g})$ be the algebra with generators $F_{i}, K_{i}, K_{i}^{-1}, 1 \leq i \leq$ $t$ and relations (4.19) and

$$
\begin{aligned}
& K_{i} F_{j} K_{i}^{-1}=q^{-d_{i} a_{i j}} F_{j} \text { for all } i, j, \\
& \sum_{r=0}^{1-a_{i j}}(-1)^{r}\left[\begin{array}{c}
1-a_{i j} \\
r
\end{array}\right]_{q_{i}} F_{i}^{1-a_{i j}-r} F_{j} F_{i}^{r}=0 \text { for all } i \neq j .
\end{aligned}
$$

The algebra $U$ is a Hopf algebra with comultiplication

$$
\Delta\left(K_{i}\right)=K_{i} \otimes K_{i}, \Delta\left(F_{i}\right)=1 \otimes F_{i}+F_{i} \otimes K_{i}^{-1} \text { for all } i .
$$

We define

$$
a_{i}=E_{i}, g_{i}=K_{i}, \text { and } \chi_{i}\left(K_{j}\right)=q^{d_{i} a_{i j}} \text { for all } i, j .
$$

Similarly, let

$$
u_{i}=F_{i} K_{i}, y_{i}=K_{i} \text {, and } \eta_{i}\left(K_{j}\right)=q^{-d_{i} a_{i j}} \text { for all } i, j .
$$

Then all the conditions (4.7), (4.8) and (4.9) for $A$ and (4.10), (4.11) and (4.12) for $U$ are satisfied.

Let $\lambda_{1}, \ldots, \lambda_{t}$ be arbitrary elements in $k$, and $\lambda=\left(\lambda_{1}, \ldots, \lambda_{t}\right)$. For all $i$, define an algebra map

$$
\gamma_{i}: A \rightarrow k \text { with } \gamma_{i}\left(E_{j}\right)=0 \text { and } \gamma_{i}\left(K_{j}\right)=\eta_{i}\left(K_{j}\right) \text { for all } j,
$$

and an $\left(\varepsilon, \gamma_{i}\right)$-derivation

$$
\delta_{i}^{\lambda}: A \rightarrow k \text { with } \delta_{i}^{\lambda}\left(E_{j}\right)=\delta_{i j} \lambda_{i} \text { and } \delta_{i}^{\lambda}\left(K_{j}\right)=\delta_{i}^{\lambda}\left(K_{j}^{-1}\right)=0 \text { for all } j .
$$


Here, $\delta_{i j}$ is the Kronecker $\delta$. Finally we define a Hopf algebra homomorphism

$$
\varphi^{\lambda}: U \rightarrow\left(A^{0}\right)^{\mathrm{cop}} \text { by } \varphi^{\lambda}\left(u_{i}\right)=\delta_{i}^{\lambda} \text { and } \varphi^{\lambda}\left(K_{i}\right)=\gamma_{i} \text { for all } i .
$$

The arguments in the proof of [AS, Lemma 5.19] show the existence of the algebra maps $\gamma_{i}$, the skew-derivations $\delta_{i}^{\lambda}$ and the Hopf algebra $\operatorname{map} \varphi^{\lambda}$.

Let $\tau^{\lambda}$ and $\sigma^{\lambda}$ be the corresponding skew pairing and 2-cocycle, and $H^{\lambda}=(U \otimes A)^{\sigma^{\lambda}}$. The subgroup $G$ generated by all $K_{i} \otimes K_{i}^{-1}$ is central in $H^{\lambda}$ by Lemma 4.3. The quotient Hopf algebra

$$
U_{q}^{\lambda}(\mathfrak{g})=H^{\lambda} /(k[G])^{+} H^{\lambda}
$$

is generated as an algebra by elements $E_{i}, F_{i}, K_{i}, K_{i}^{-1}, 1 \leq i \leq t$, with relations (4.19), (4.20), (4.21), (4.23), (4.24), together with

$$
E_{i} F_{j}-F_{j} E_{i}=\delta_{i j} \lambda_{i}\left(K_{i}^{-1}-K_{i}\right) \text { for all } i, j .
$$

This can be seen in the same way as in the end of the proof of $\mathrm{AS}$, Theorem 5.17]. Note that the relations (4.26) are a special case of the relations (4.18). The comultiplication is defined by (4.22), (4.25). The group $G\left(U_{q}^{\lambda}(\mathfrak{g})\right)$ is the free abelian group with basis $K_{1}, \ldots, K_{t}$.

Since by definition, $U_{q}(\mathfrak{g})=U_{q}^{\lambda}(\mathfrak{g})$, where $\lambda_{i}=q^{-d_{i}}-q^{d_{i}}$ for all $i$, we obtain the following.

Theorem 4.5. Let $G=G\left(U_{q}(\mathfrak{g})\right)$. Then for any $k$-algebra $B$, the inclusion $\iota: k[G] \rightarrow U_{q}(\mathfrak{g})$ induces a bijective map

$$
\mathcal{H}_{B}\left(U_{q}(\mathfrak{g})\right) \cong \mathcal{H}_{B}(k[G]) .
$$

Proof. If we take $\lambda_{i}=0$ for all $i$, then $U_{q}^{\lambda}(\mathfrak{g})$ is a graded Hopf algebra. For another choice of $\lambda$ we get $U_{q}(\mathfrak{g})$. Hence by Proposition 4.4. $U_{q}(\mathfrak{g})$ is a 2-cocycle twist of a graded Hopf algebra with the group algebra $k[G]$ as degree 0 part, and the claim follows from Corollary 2.11.

Remark 4.6. Let $q$ be a primitive $N$-th root of unity, and assume that $N$ is odd and not divisible by 3 if the Dynkin diagram of $\mathfrak{g}$ contains a component $G_{2}$. The finite-dimensional Frobenius-Lusztig kernel $u_{q}(\mathfrak{g})$ is the quotient Hopf algebra of $U_{q}(\mathfrak{g})$ defined by adding the relations saying that the $N$-th powers of the positive root vectors be 0 (see [CP. p. 120], [AJS, p. 16]). By [AS, Lemma 5.24] the above Hopf algebra homomorphism $\varphi^{\lambda}$ also exists for $u_{q}(\mathfrak{g})$. This implies that $u_{q}(\mathfrak{g})$ is a 2-cocycle twist of a graded Hopf algebra. Hence we have a bijection

$$
\mathcal{H}_{B}\left(u_{q}(\mathfrak{g})\right) \rightarrow \mathcal{H}_{B}(k[G]),
$$

where $G=G\left(u_{q}(\mathfrak{g})\right) \cong\left(C_{N}\right)^{t}$. 
There are numerous other Hopf algebras that are 2-cocycle twists of graded Hopf algebras (see Ma2, and Di for all the Hopf algebras introduced in $\mathrm{AS}$ ). We do not know any example of pointed Hopf algebras not of this type.

\section{REFERENCES}

[AJS] H. H. Andersen, J. Jantzen, and W. Soergel, Representations of quantum groups at a $p$-th root of unity and of semisimple groups in characteristic $p$ : Independence of $p$, Astérisque 220, Soc. Math. France, Paris, 1994.

[AS] N. Andruskiewitsch and H.-J. Schneider, Finite quantum groups over abelian groups of prime exponent, Ann. Sci. Ec. Norm. Supér. 35 (2002), 1-26.

[B] H. Bass, Algebraic K-theory, Benjamin, New York, 1968.

[BH] T. Brzeziński and P. M. Hajac, Galois type extensions and non-commutative geometry, preprint 2003.

[C] S. Caenepeel, Brauer Groups, Hopf Algebras and Galois Theory, Kluwer Acad. Publ., Dordrecht, 1998.

[CP] C. de Concini and C. Procesi, Quantum Groups, in "D-modules, Representation Theory and Quantum Groups", 31-140, Lecture Notes in Mathematics 1565, Springer-Verlag, Berlin, 1993.

[Di] D. Didt, Linkable Dynkin diagrams and quasi-isomorphisms for finite dimensional pointed Hopf algebras, $\mathrm{PhD}$ thesis, Ludwig-Maximilians-Universität München, 2002.

[D] Y. Doi, Braided bialgebras and quadratic bialgebras, Comm. Algebra 17 (1989), 3053-3085.

[DT] Y. Doi and M. Takeuchi, Multiplication alteration by two-cocycles. The quantum version, Comm. Algebra 22 (1994), 5715-5732.

[G] S. M. Gersten, On Mayer-Vietoris functors and algebraic K-theory, J. Algebra 18 (1971), 51-88.

[Ha] H. Hasse, Die Multiplikationsgruppe der abelschen Körper mit fester GaloisGruppe, Abh. Math. Sem. Univ. Hamburg 16 (1949), 29-40.

[Hu] D. Husemoller, Fibre Bundles, Second Edition, Grad. Texts in Math. 20, Springer-Verlag, New York-Heidelberg, 1975.

[J] J. C. Jantzen, Lectures on Quantum Groups, Graduate Studies in Mathematics, Volume 6, Amer. Math. Soc., Providence, RI, 1995.

[K] C. Kassel, Quantum principal bundles up to homotopy equivalence, in Proc. Abel Centennial Conference, The legacy of Niels Henrik Abel, O. A. Laudal, R. Piene (eds), Springer-Verlag 2003.

[KS] A. Klimyk and K. Schmüdgen, Quantum Groups and Their Representations, Texts and Monographs in Physics, Springer-Verlag, Berlin, 1997.

[KT] H. F. Kreimer and M. Takeuchi, Hopf algebras and Galois extensions of an algebra, Indiana Univ. Math. J. 30 (1981), 675-692.

[L] T. Y. Lam, Lectures on modules and rings, Grad. Texts in Math. 189, SpringerVerlag, New York, 1999.

[Ma1] A. Masuoka, Cleft extensions for a Hopf algebra generated by a nearly primitive element, Comm. Algebra 22 (1994), 4537-4559.

[Ma2] A. Masuoka, Defending the negated Kaplansky conjecture, Proc. Amer. Math. Soc. 129 (2001), 3185-3192. 
[M] S. Montgomery, Hopf Algebras and Their Actions on Rings, CBMS Conf. Series in Math., vol. 82, Amer. Math. Soc., Providence, RI, 1993.

[MS] S. Montgomery and H.-J. Schneider, Prime ideals and twisting in Hopf Galois extensions, preprint 2003.

[P] C. Pedrini, On the $K_{0}$ of certain polynomial extensions, in "Algebraic $K$ theory, II: Classical algebraic $K$-theory and connections with arithmetic" (Proc. Conf., Battelle Memorial Inst., Seattle, Wash., 1972), 92-108, Lecture Notes in Mathematics 342, Springer-Verlag, Berlin, 1973.

[Sch] P. Schauenburg, Hopf-Galois and bi-Galois extensions, preprint (2003).

[S] H.-J. Schneider, Principal homogeneous spaces for arbitrary Hopf algebras, Israel J. Math. 72 (1990), 167-195.

[Sw1] R. G. Swan, Some relations between higher K-functors, J. Algebra 21 (1972), 113-136.

[Sw2] R. G. Swan, On seminormality, J. Algebra 67 (1980), 210-229.

[T] M. Takeuchi, On extensions of formal groups by $\mu^{A}$, Comm. Algebra 5 (1977), 1439-1481.

Christian Kassel: Institut de Recherche Mathématique Avancée, CNRS-Université Louis Pasteur, 7 rue René Descartes, 67084 StrasBOURG, FRANCE

E-mail address: kassel@math.u-strasbg.fr

Hans-JÜrgen Schneider: Mathematisches Institut, Universität MünChen, Theresienstr. 39, D-80333 Munich, Germany

E-mail address: Hans-Juergen.Schneider@mathematik. uni-muenchen.de 